Viktor Hofmann* and Jens Twiefel

\title{
Optimization of a Piezoelectric Bending Actuator for a Tactile Virtual Reality Display
}

\begin{abstract}
The excitation of mechanoreceptors in the finger with different frequencies and intensities generates a tactile impression. For the experience of a complete surface many distributed sources are needed in the tactile display. For these local stimulations of the finger several piezoelectric bending actuators will be arranged in an array perpendicular to the skin. The challenge in the system design is to transfer high dynamic shear forces to the skin at required frequencies together with a compact display design. In order to estimate the dynamic behavior of the bending actuators a transfer matrix method model based on the Timoshenko beam theory is derived. Beside the outer geometric values, the layered structure of the actuator is included in the model. In addition the influence of the load on the actuator's tip in lateral and in normal direction as well as on the rotational degree of freedom is taken into account. Using the analytical approach, a parametric study is carried out to find an optimized actuator design for the display. For the validation, the modeled beam is compared with experimental data.
\end{abstract}

Keywords: tactile display, loaded piezoelectric beam, transfer matrix method, multi-layer structure, Timoshenko

DOI 10.1515/ehs-2014-0055

\section{Introduction}

The quality of today's visual and acoustic presentation requires little improvement even on home and office computers. Haptic impressions are limited usually only to the contours of keyboard and mouse. Our goal is to

\footnotetext{
*Corresponding author: Viktor Hofmann, Institute of Dynamics and Vibration Research, Gottfried Wilhelm Leibniz University of Hannover, Hannover, Germany, E-mail: hofmann@ids.uni-hannover.de

Jens Twiefel, Institute of Dynamics and Vibration Research, Gottfried Wilhelm Leibniz University of Hannover, Hannover, Germany,

E-mail: twiefel@ids.uni-hannover.de
}

develop a tactile virtual reality display, which allows tactual perception of different virtual surface structures such as wood, leather, plastic and many more. For tactile perception a variety of different mechanoreceptors located in the finger are responsible. Each type of receptor is particularly sensitive to certain stimulations and excitation frequencies. There are receptors that are specialized in the detection of skin shearing, while others sense perpendicular skin deformations. In order to recreate tactile surface impressions as accurately as possible, a selective excitation of these different receptors must be done.

The perception of textures and roughness needs the application of tangential forces on the skin (Hatzfeld, 2013); therefore, aside from normal forces the final version of the tactile display will generate shear forces. The generation of skin shearing will be realized by piezoelectric bending actuators. Hence for the description of the actuator behavior an analytic actuator model in the form of a transfer matrix is established and the contact loads, which are generated from the finger, are measured and translated into a load model. Based on the experimentally supported actuator-load model the frequencydependent actuator behavior under operating load can be estimated and the actuator design can be optimized. For the necessary skin stretching the actuator tip displacements and forces have to be as high as possible, whereas the sizing has to be preferable small to achieve high spatial resolution and a compact design. The tactile perception focus of the fingers is up to $1 \mathrm{kHz}$ (Kern et al. 2009), which will be the working area of the display.

\section{Actuator Model}

To model the behavior of the bending actuator the transfer matrix method as described by Pestel et al. (1963) is applied. Under this method, the relationship between the state variables of the actuator is described by a matrix, which is derived from an analytical model. Thus, the method offers, compared to a finite element 
method (FEM) calculation, a reduced computation time and a simple way to combine several passive and active elements by matrix multiplication. To apply the method to our application, it is necessary to derivate a transfer matrix for the used piezoelectric bending actuator.

For the tactile display an actuator with two parallel connected piezoelectric layers is to be used. The modeling of the bimorph is based on the Timoshenko beam theory and is similar to the modeling of Cho et al. (2000) for a bimorph with serial connected piezoelectric layers based on the Euler-Bernoulli beam theory. In contrast to the Euler-Bernoulli theory the Timoshenko theory considers the shear deformation and the rotary inertia. As a result, the Timoshenko theory is more suitable for beams with a small length to cross-section ratio and high frequencies (Dietl et al. 2010).

In the presented modeling steady-state, harmonic vibrations with the excitations frequency $\omega$ are assumed. Therefore, the state variables and the boundary conditions can be expressed as complex amplitudes.

\section{Analytical Model of a Piezoelectric Parallel Bimorph for Steady-State, Harmonic Vibrations}

\section{Bimorph Description}

The examined piezoelectric bimorph consists of two identical piezoelectric layers (height $h_{\mathrm{p}}$ ) and one conductive passive middle shim layer (height $h_{\mathrm{s}}$ ). The piezoelectric layers have the same polarization direction $P$ and are driven in an electrical parallel connection (parallel bimorph); see Figure 1. An applied electric potential leads to an extension of the one and a shortening of the other piezoelectric layer, which results in an actuator bending. The state variables of the actuator are the electric values - voltage $U$ and current $I$ - and the mechanical values - velocity $v$, angular velocity $\dot{\psi}$, moment $M$ and force $F$ - for each end of the bimorph.

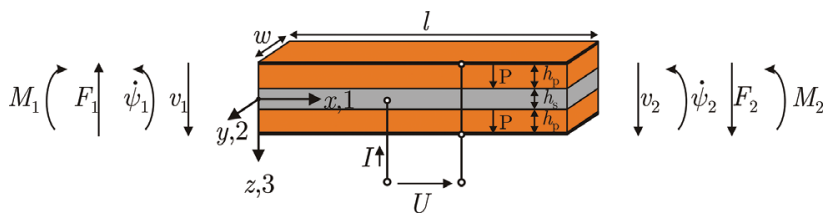

Figure 1: Geometry and boundary values of a bimorph.
To describe the material behavior of the middle shim (indicated by the index $s$ ) the Hooke's law is sufficient:

$$
S_{1, s}=s_{11, s} T_{1, s} \quad S_{5, s}=S_{55, s} T_{5, s}
$$

Whereas in the description of the material behavior of the piezoelectric layers (index $p$ ) the following piezoelectric material laws, which apply only to small mechanical and electrical amplitudes, are adopted (Dietl et al. 2010; Standards Committee of the IEEE Ultrasonics 1987):

$$
\begin{aligned}
& S_{1, p}=s_{11}^{\mathrm{E}} T_{1, p}+d_{31} E_{3} \quad S_{5, p}=s_{55}^{\mathrm{E}} T_{5, p} \\
& D_{3, p}=d_{31} T_{1, p}+\varepsilon_{33}^{\mathrm{T}} E_{3}
\end{aligned}
$$

The linear dependences between mechanical strain $S_{1}$, mechanical stress $T_{1}$, shear $S_{5}$, shear stress $T_{5}$, dielectric charge displacement $D_{3}$ and electrical field strength $E_{3}$ are described by the elastic compliance $s_{11}$, the shear compliance $s_{55}$, the piezoelectric constant $d_{31}$ and the dielectric constant $\varepsilon_{33}$. The superscript $\mathrm{E}$ indicates constant electric field conditions and $\mathrm{T}$ constant stress conditions for piezoelectric materials. The electrical field strength $E_{3}$ for the parallel bimorph can also be expressed as a function of voltage $U$ :

$$
E_{3}=\frac{U}{h_{p}}
$$

\section{Kinematics of the Timoshenko Beam}

Figure 2 illustrates the kinematics of a Timoshenko beam in the $x z$-plane.

$\varphi$ is the beam tilting and $\psi$ the rotation angle of the cross section. The displacement $u_{z}$ in the $z$-direction corresponds to the beam deflection curve. In contrast to the displacement $u_{x}$ in axial direction it is independent of the coordinate $z$ for the linearized case. With $\frac{\partial(*)}{\partial x}=(*)^{\prime}$ the linearized kinematic relations can be summarized as follows:

$$
\begin{aligned}
& \varphi(x)=u_{z}^{\prime} \quad \psi(x)=\frac{\partial u_{x}}{\partial z} \\
& u_{x}(x, z)=z \psi \quad u_{z}(x)
\end{aligned}
$$

The beam shearing and the strain in the $\mathrm{x}$-direction, which are caused by the bending, are given by

$$
S_{1}=u_{x}^{\prime}=\psi^{\prime} z \quad S_{5}=\varphi+\psi
$$




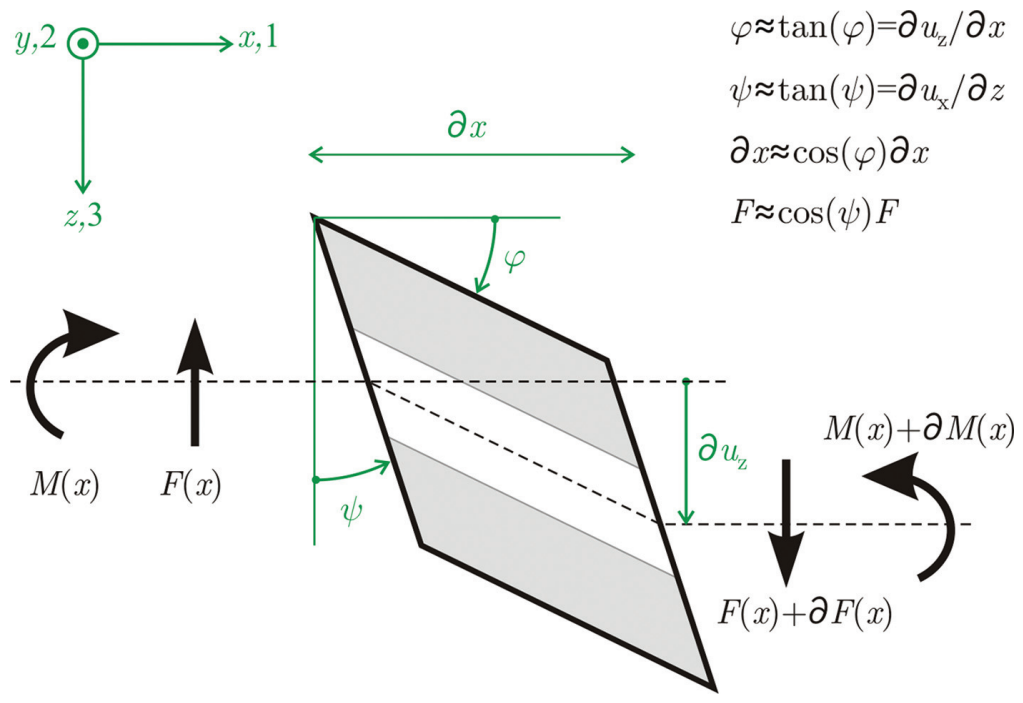

Figure 2: Linearized kinematics of the Timoshenko bimorph.

Simplified, in this consideration an equal shear in all layers and an ideal composite without sliding of the individual layers on each other is assumed.

\section{Derivation of the State Variables}

According to Figure 2 the force and moment balance at the infinitesimally short beam leads to the following relationships:

$$
\begin{aligned}
\frac{\partial M}{\partial x} & =F-\omega^{2} \psi\left[\int_{A_{\mathrm{s}}} \rho_{\mathrm{s}} z^{2} d A+2 \int_{A_{\mathrm{p}}} \rho_{\mathrm{p}} z^{2} d A\right] \\
& =F-\omega^{2} \psi \underbrace{\left[\rho_{s} w \frac{h_{s}^{3}}{12}+\rho_{p} w \frac{2}{3}\left(\left(h_{p}+\frac{h_{s}}{2}\right)^{3}-\left(\frac{h_{s}}{2}\right)^{3}\right)\right]}_{(\rho I)_{\mathrm{eff}}}
\end{aligned}
$$

$$
\frac{\partial F}{\partial x}=-\omega^{2} u_{z} \underbrace{w\left(\rho_{s} h_{s}+2 \rho_{p} h_{p}\right)}_{\hat{m}_{\mathrm{eff}}}
$$

Here, $F$ is the linearized lateral force (see Figure 2 ) and $\hat{m}_{\text {eff }}$ the effective mass distribution of the bimorph, which is composed of the beam width $w$ and the density $\rho$ of the respective layer. $M$ describes the inner bending moment and the abbreviation $(\rho I)_{\text {eff }}$ the effective moment of inertia with the cross section of the middle shim layer $A_{\mathrm{s}}$ and the cross section of one piezoelectric layer $A_{\mathrm{p}}$.

In addition the bending moment $M$ can be determined from the axial stress and the shear force $F$ from the shear stress, which are acting in the cross-sections:

$$
\begin{aligned}
& M=\int_{A_{\mathrm{s}}} T_{1, \mathrm{~s}} z d A+2 \int_{A_{\mathrm{p}}} T_{1, \mathrm{p}} z d A \\
& =\underbrace{\left[\frac{w}{s_{11, s}} \frac{h_{s}^{3}}{12}+\frac{w}{s_{11}^{E}} \frac{2}{3}\left(h_{\mathrm{p}}+\left(\frac{h_{s}}{2}\right)^{3}-\left(\frac{h_{s}}{2}\right)^{3}\right)\right]}_{(E I)_{\mathrm{eff}}} \psi^{\prime} \\
& -\underbrace{\left[\frac{w d_{31}}{s_{11}^{E}}\left(h_{s}+h_{p}\right)\right]}_{K} U \\
& F=\kappa^{2} \int_{A_{\mathrm{s}}} T_{5, \mathrm{~s}} d A+2 \kappa^{2} \int_{A_{\mathrm{p}}} T_{5, \mathrm{p}} d A \\
& =\kappa^{2} w\left[\frac{h_{s}}{s_{55, s}}+\frac{2 h_{p}}{s_{11}^{\mathrm{E}}}\right]\left(u_{z}^{\prime}+\psi\right) \\
& (A G)_{\mathrm{eff}}
\end{aligned}
$$

The stresses $T_{1, \mathrm{~s}}, T_{1, \mathrm{p}}, T_{5, \mathrm{~s}}$ and $T_{5, \mathrm{p}}$ are derived from eqs [1][5]. For clarity the constants $(E I)_{\mathrm{eff}},(A G)_{\mathrm{eff}}$ and $K$ are introduced. $(E I)_{\text {eff }}$ corresponds to the effective bending stiffness and $(A G)_{\text {eff }}$ is the effective shear stiffness of the bimorph. $(A G)_{\text {eff }}$ includes the correction factor $\kappa^{2}$, which depends only on the shape of the beam cross section and has for rectangular cross section usually the value 5/6 (Gasch et al. 2012).

The velocity $v$ in $z$-direction results from the timederived displacement:

$$
v=j \omega u_{z}
$$

To calculate the rotation velocity of the cross section the time derivation of eq. [10] can be used:

$$
\dot{\psi}=\frac{j \omega F}{(A G)_{\mathrm{eff}}}-j \omega u_{z}^{\prime}
$$


The determination of the electric current $I$ requires an integration of the time-derived dielectric charge displacement $\dot{D}_{3}$ over the electrode surface $A_{\mathrm{e}}=w l$ of the upper and lower piezoelectric layers (Standards Committee of the IEEE Ultrasonics 1987):

$$
\begin{aligned}
& I(t)=\int_{A_{\mathrm{e}}} \dot{D}_{3, \text { low }}-\dot{D}_{3, \text { up }} d A_{\mathrm{e}} \\
& =\int_{0}^{l} D(\underbrace{\left(\frac{1}{s_{11}^{\mathrm{E}}}\left(\frac{h_{s}+h_{p}}{2}\right) \dot{\psi}^{\prime}-\frac{d_{31}}{s_{11}^{\mathrm{E}} h_{p}} \dot{U}\right)}_{T_{1, \mathrm{~m}}}+\frac{\varepsilon_{33}^{T}}{h_{p}} \dot{U}) d x \\
& -w \int_{0}^{l}\left(d_{31}^{\left(\frac{-1}{s_{11}^{\mathrm{E}}}\left(\frac{h_{s}+h_{p}}{2}\right) \dot{\psi}^{\prime}+\frac{d_{31}}{s_{11}^{\mathrm{E}} h_{p}} \dot{U}\right)}-\frac{\varepsilon_{33}^{T}}{h_{p}} \dot{U}\right) d x \\
& =\underbrace{\left[\frac{2 w l}{h_{p}}\left(\varepsilon_{33}^{T}-\frac{d_{31}^{2}}{s_{11}^{E}}\right)\right]}_{C_{\mathrm{p}}} \dot{U} \\
& +\underbrace{\left[\frac{w d_{31}\left(h_{s}+h_{p}\right)}{s_{11}^{\mathrm{E}}}\right]}_{K}[\dot{\psi}(x=l)-\dot{\psi}(x=0)]
\end{aligned}
$$

In the calculation of the current, the direction of polarization $P$ in the electric circuit and the direction of axial stress $T_{1}$ in the single layers must be considered. In eq. [12] the absolute value of the mean stress $T_{1, \mathrm{~m}}$ is adopted. This stress acts in the middle of both piezoelectric layers in opposite directions.

The introduced abbreviation $C_{p}$ corresponds to the capacity of the entire bimorph and with the factor $K$ the electrical dynamic is coupled to the rotational dynamics.

\section{Computation of the Transfer Matrix}

With some substitutions and differentiations of the eqs [6]-[9] following fourth-order differential equation of motion results:

$$
\begin{aligned}
u_{z}^{\prime \prime \prime \prime}+ & u_{z}^{\prime \prime} \underbrace{\omega^{2}\left(\frac{(\rho I)_{\mathrm{eff}}}{(E I)_{\mathrm{eff}}}+\frac{\hat{m}_{\mathrm{eff}}}{(A G)_{\mathrm{eff}}}\right)}_{n_{1}} \\
& +u_{z} \underbrace{\frac{\omega^{2} \hat{m}_{\mathrm{eff}}}{(E I)_{\mathrm{eff}}}\left(\frac{(\rho I)_{\mathrm{eff}}}{(A G)_{\mathrm{eff}}}-1\right)}_{n_{2}}=0
\end{aligned}
$$

This equation corresponds to the known form of a differential equation for the passive Timoshenko beam and has the following eigenvalues; compare (Pestel et al. 1963):

$$
\lambda_{1,2}=\sqrt{\sqrt{\frac{1}{4} n_{1}^{2}-n_{2}} \mp \frac{1}{2} n_{1}}
$$

Based on the differential equation, the sought state variables for a harmonic excitation can be determined similar to a passive beam as described by Pestel et al. (1963) with the following approach for the force:

$$
\begin{aligned}
F= & a_{1} \cosh \left(\frac{\lambda_{1} x}{l}\right)+a_{2} \sinh \left(\frac{\lambda_{1} x}{l}\right) \\
& +a_{3} \cos \left(\frac{\lambda_{2} x}{l}\right)+a_{4} \sin \left(\frac{\lambda_{2} x}{l}\right)
\end{aligned}
$$

With this approach results from eqs [10] and [7]:

$$
\begin{aligned}
v= & \frac{1}{j \omega \hat{\mathrm{m}}_{\mathrm{eff}}}\left(a_{1} \frac{\lambda_{1}}{l} \sinh \left(\frac{\lambda_{1} x}{l}\right)+a_{2} \frac{\lambda_{1}}{l} \cosh \left(\frac{\lambda_{1} x}{l}\right)\right. \\
& \left.-a_{3} \frac{\lambda_{2}}{l} \sin \left(\frac{\lambda_{2} x}{l}\right)+a_{4} \frac{\lambda_{2}}{l} \cos \left(\frac{\lambda_{2} x}{l}\right)\right)
\end{aligned}
$$

Equation [11] and the previously determined eqs [15] and [16] provide:

$$
\begin{aligned}
\dot{\psi}= & j \omega\left(\frac{1}{(A G)_{\mathrm{eff}}}+\frac{1}{\omega^{2} \hat{m}_{\mathrm{eff}}} \frac{\lambda_{1}^{2}}{l^{2}}\right)\left(a_{1} \cosh \left(\frac{\lambda_{1} x}{l}\right)+a_{2} \sinh \left(\frac{\lambda_{1} x}{l}\right)\right) \\
& +j \omega\left(\frac{1}{(A G)_{\mathrm{eff}}}-\frac{1}{\omega^{2} \hat{m}_{\mathrm{eff}}} \frac{\lambda_{2}^{2}}{l^{2}}\right)\left(a_{3} \cos \left(\frac{\lambda_{2} x}{l}\right)+a_{4} \sin \left(\frac{\lambda_{2} x}{l}\right)\right)
\end{aligned}
$$

Equations [8] and [17] give

$$
\begin{aligned}
M= & \left(\frac{(E I)_{\mathrm{eff}}}{(A G)_{\mathrm{eff}}}+\frac{(E I)_{\mathrm{eff}}}{\omega^{2} \hat{m}_{\mathrm{eff}}} \frac{\lambda_{1}^{2}}{l^{2}}\right) \frac{\lambda_{1}}{l} \\
& \left(a_{1} \sinh \left(\frac{\lambda_{1} x}{l}\right)+a_{2} \cosh \left(\frac{\lambda_{1} x}{l}\right)\right) \\
& -\left(\frac{(E I)_{\mathrm{eff}}}{(A G)_{\mathrm{eff}}}-\frac{(E I)_{\mathrm{eff}}}{\omega^{2} \hat{m}_{\mathrm{eff}}} \frac{\lambda_{2}^{2}}{l^{2}}\right) \frac{\lambda_{2}}{l} \\
& \left(a_{3} \sin \left(\frac{\lambda_{2} x}{l}\right)-a_{4} \cos \left(\frac{\lambda_{2} x}{l}\right)\right)-K U
\end{aligned}
$$

And the solution for the current is

$$
\begin{aligned}
I= & j \omega C_{\mathrm{p}} U+j \omega K\left[\left(\frac{1}{(A G)_{\mathrm{eff}}}+\frac{1}{\omega^{2} \hat{m}_{\mathrm{eff}}} \frac{\lambda_{1}^{2}}{l^{2}}\right)\right. \\
& \left(a_{1} \cosh \left(\lambda_{1}\right)+a_{2} \sinh \left(\lambda_{1}\right)\right) \\
& +\left(\frac{1}{(A G)_{\mathrm{eff}}}-\frac{1}{\omega^{2} \hat{m}_{\mathrm{eff}}} \frac{\lambda_{2}^{2}}{l^{2}}\right)\left(a_{3} \cos \left(\lambda_{2}\right)+a_{4} \sin \left(\lambda_{2}\right)\right) \\
& \left.-\left(\frac{a_{1}+a_{3}}{(A G)_{\mathrm{eff}}}+\frac{a_{1} \lambda_{1}^{2}-a_{3} \lambda_{2}^{2}}{\omega^{2} \hat{m}_{\mathrm{eff}} l^{2}}\right)\right]
\end{aligned}
$$


As described by Gasch, Knothe, and Liebich (2012), the unknown coefficients $a_{1}, a_{2}, a_{3}$ and $a_{4}$ can be determined with the complex boundary conditions $\left[\begin{array}{lllll}v_{1} & \dot{\psi}_{1} & M_{1} & F_{1} & U\end{array}\right]^{T}$ and then used to calculate $\left[\begin{array}{lllll}v_{2} & \dot{\psi}_{2} & M_{2} & F_{2} & I\end{array}\right]^{T}$. So from the resulting system of equations the transfer matrix can be set $\mathrm{p}$ as follows: which in the model can be taken into account by a complex Young's modulus or a complex elastic compliance for the shim and the piezoelectric layers (Sollmann, 1981):

$$
\frac{1}{s_{11}^{* \mathrm{E}}}=\frac{1}{s_{11}^{\mathrm{E}}}\left(1+j \frac{1}{Q_{\mathrm{p}}}\right) \frac{1}{s_{11, \mathrm{~s}}^{*}}=\frac{1}{s_{11, \mathrm{~s}}}\left(1+j \frac{1}{Q_{\mathrm{s}}}\right)
$$

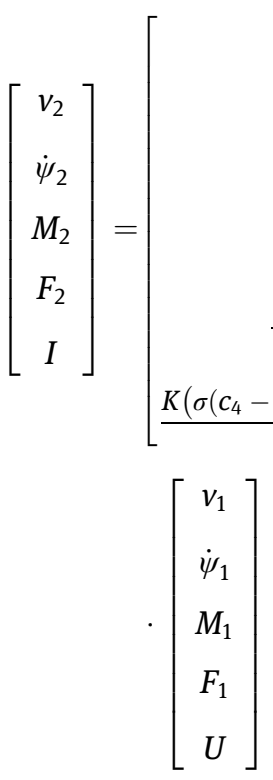

with

$$
\begin{array}{ll}
c_{0}=\frac{\lambda_{2}^{2} \cosh \left(\lambda_{1}\right)+\lambda_{1}^{2} \cos \left(\lambda_{2}\right)}{\lambda_{1}^{2}+\lambda_{2}^{2}} & c_{1}=\frac{\frac{\lambda_{2}^{2}}{\lambda_{1}} \sinh \left(\lambda_{1}\right)+\frac{\lambda_{1}^{2}}{\lambda_{2}} \sin \left(\lambda_{2}\right)}{\lambda_{1}^{2}+\lambda_{2}^{2}} \\
c_{2}=\frac{\cosh \left(\lambda_{1}\right)-\cos \left(\lambda_{2}\right)}{\lambda_{1}^{2}+\lambda_{2}^{2}} & c_{3}=\frac{\frac{1}{\lambda_{1}} \sinh \left(\lambda_{1}\right)-\frac{1}{\lambda_{2}} \sin \left(\lambda_{2}\right)}{\lambda_{1}^{2}+\lambda_{2}^{2}} \\
c_{4}=\frac{\lambda_{1} \sinh \left(\lambda_{1}\right)+\lambda_{2} \sin \left(\lambda_{2}\right)}{\lambda_{1}^{2}+\lambda_{2}^{2}} & c_{5}=\frac{\lambda_{1}^{2} \sinh \left(\lambda_{1}\right)+\lambda_{2}^{2} \sin \left(\lambda_{2}\right)}{\lambda_{1}^{2}+\lambda_{2}^{2}} \\
\sigma=\frac{\hat{m}_{\mathrm{eff}} \omega^{2}}{(G A)_{\mathrm{eff}}} \quad \tau=\frac{(\rho I)_{\mathrm{eff}} \omega^{2} l^{2}}{(G A)_{\mathrm{eff}}} \quad a=\frac{l^{2}}{(E I)_{\mathrm{eff}}} \beta^{4}=\frac{\hat{m}_{\mathrm{eff}} \omega^{2} l^{4}}{(E I)_{\mathrm{eff}}}
\end{array}
$$

Aside from the additional fifth row and the fifth column with the electromechanical matrix elements, the matrix corresponds to the transfer matrix of a passive bending beam (compare Pestel et al. (1963)).

\section{Damping}

In the previous calculations, the damping of the system was not taken into account. When exciting the bimorph many factors lead to losses. One of these is the material damping,
The quality factor $Q$ is a characteristic material parameter. Other mechanical damping effects, such as the losses due to local friction in the bearing or the friction with the surrounding air, are difficult to determine separately, but in the model these effects can be considered by decreasing $Q$.

In addition to the mechanical damping, there are dielectric and piezoelectric losses in the piezoelectric material. Equivalent to the complex modulus, the dielectric loss can be taken into account by a complex dielectric constant $\varepsilon_{33}^{* \mathrm{~T}}$ with the dissipation factor $\tan \delta$ and the piezoelectric loss by a complex piezoelectric constant $d_{31}^{*}$ with the dissipation factor $\tan \theta$ (Uchino et al. 2001):

$$
\varepsilon_{33}^{* \mathrm{~T}}=\varepsilon_{33}^{\mathrm{T}}(1+j \tan \delta) \quad d_{31}^{*}=d_{31}(1+j \tan \theta)
$$

\section{Validation of the Actuator Model}

The validation of the actuator model occurred by using a bending actuator from Johnson Matthey consisting of the piezoelectric material "VIBRIT 1100" (Johnson Matthey Piezo Products $\mathrm{GmbH}$ 2014) with a carbon fiber-reinforced polymer middle shim layer. The properties of the 


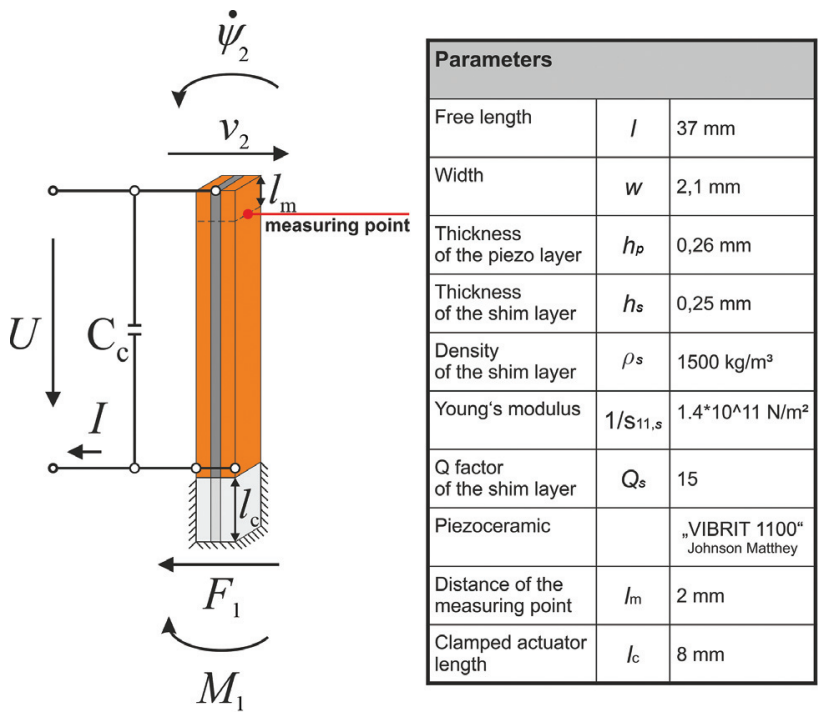

Figure 3: Model parameters of the bending actuator.

intermediate layer are adopted as shown in Figure 3. The operating voltage during the measurement was $1 \mathrm{~V} \mathrm{AC}$ and the boundary conditions were fixed free.

For model validation, the mechanical and electrical admittance $Y_{\text {mech }}$ and $Y_{\mathrm{el}}$ of the real and modeled system are compared. Therefore the velocity was measured with a laser vibrometer and the current with a current clamp.

For accurate modeling a consideration of the existing measurement conditions is required. Among others, this includes the position of the velocity measuring point. Since depending on the position some mode shapes have a stronger influence on the velocity measurement than other. The transfer matrix method enables an easy consideration of the measuring point by splitting the actuator into two pieces. So the measured values can be compared with the state variables at the intersection.

Furthermore, the modeled system damping has to be adapted to the measurement, otherwise the modeled amplitude is not correct, especially in the resonance. This is done by adapting the $Q$ factor of the shim layer.

Also, the clamped actuator segment is considered by the capacity $C_{\mathrm{c}}$ (see Figure 3 ):

$$
C_{\mathrm{c}}=\left[\frac{2 w l_{c}}{h_{p}}\left(\varepsilon_{33}^{\mathrm{S}}-\frac{d_{31}^{2}}{s_{11}^{\mathrm{E}}}\right)\right]
$$

Without this step, the measured current is higher than the modeled current.

Figure 4 shows a comparison of the measurement and the model. Clearly visible is the good correlation, especially in the first mode. Thus, the model is suitable for the actuator optimization, especially because for the tactile display the first mode shape, which has the largest displacement amplitudes at the actuator end, is to be utilized.

\section{Load}

\section{Load Influence}

In order to investigate the influence of the finger on the bimorph a test assembly was setup, as illustrated in Figure 5. In this test, a subject has to place his finger pad relaxed on a $2 \mathrm{~mm} \times 3 \mathrm{~mm}$ wide opening, thereby he touches the bimorph tip and the actuator can stimulate the finger tangentially. During the entire measurement time he has to keep the applied force constant.

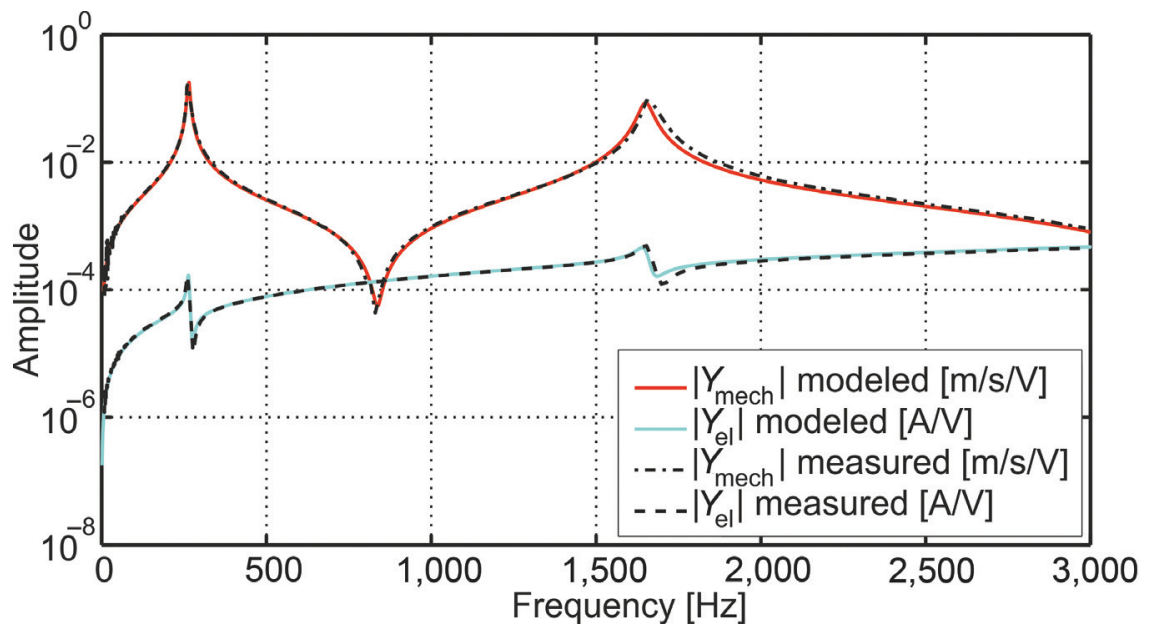

Figure 4: Comparison of measurement and model. 

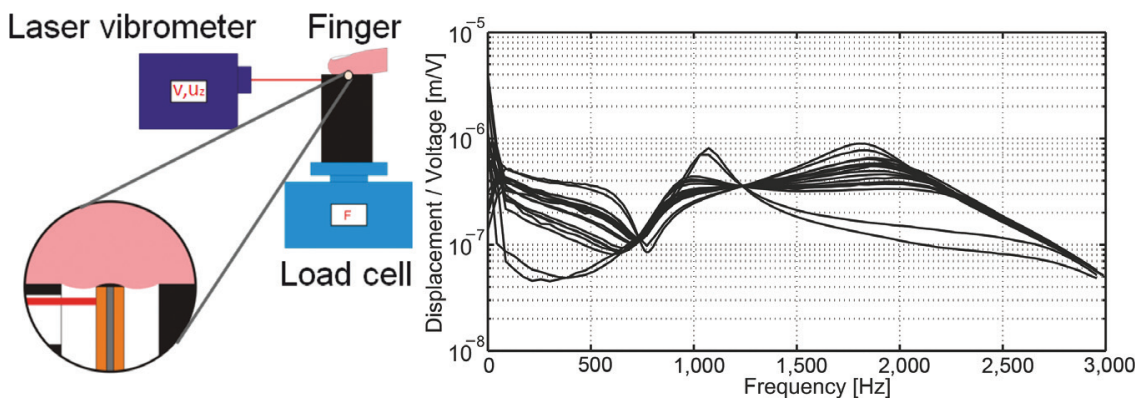

Figure 5: Displacement measuring on a loaded bimorph.

The graph in Figure 5 shows the measurements at the index and middle fingers of six subjects. It is visible, that the finger changes noticeably the system behavior and that there are strong differences between each curves. Reasons for the variations are beside the differences between the individual fingers, the influence of the pressure, position and orientation of the finger.

\section{Load Modeling}

The finger load can be divided into a tangential component in $z$-direction, the load influence on the rotational degree of freedom $\psi$ and the normal component in $x$-direction.

Since the normal force $N$ generated by the finger changes only slightly and the movement of the bimorph in $x$-direction is negligible, $N$ can be modeled as a constant force. In the transfer matrix, as described by Pestel et al. (1963), a constant normal force can be taken into account by exchanging $\tau$ with

$$
\tau^{*}=\tau-\frac{N l^{2}}{(E I)_{\mathrm{eff}}} .
$$

A model-based examination shows that the normal forces generated by the finger are normally too small $(|N|<0,1 N)$ to influence the system behavior significantly. Therefore, this load component is neglected.

For the consideration of the other load components it must be taken into account that the human tissue has viscoelastic material behavior (Kern et al. 2009). So the generalized Maxwell material model (Figure 6) is chosen as approach and the load components are included in the overall modeling as a translational impedance $Z_{\mathrm{L}}$ and a rotational impedance $Z_{\mathrm{L}, r}$ :

$$
\begin{aligned}
Z_{\mathrm{L}} & =\frac{F_{2}}{v_{2}}=\frac{k_{0}}{j \omega}+d_{0}+\sum_{i=1} \frac{k_{i} d_{i}}{k_{i}+j \omega d_{i}} \\
Z_{\mathrm{L}, r} & =\frac{M_{2}}{\dot{\psi}_{2}}=\frac{k_{\mathrm{r}, 0}}{j \omega}+d_{r, 0}+\sum_{i=1} \frac{k_{r, i} d_{r, i}}{k_{r, i}+j \omega d_{r, i}}
\end{aligned}
$$

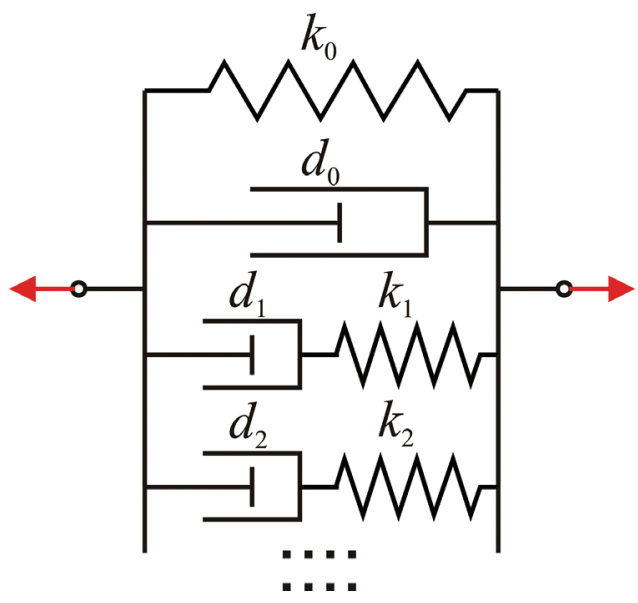

Figure 6: Generalized Maxwell model for the translational load component.

Already with two translational stiffness parameters $k_{0}$ and $k_{1}$ and two damping parameters $d_{0}$ and $d_{1}$ the measurements can be well approximated, especially in the for the application important lower frequency range. Due to the small angle changes and the small contact area between finger and actuator tip the rotational lode influence can be neglected $\left(Z_{\mathrm{L}, r}=0\right)$. This assumption is confirmed by the simulation results. Exemplarily the curves from Figure 5 with the maximal and the minimal impedances were selected and modeled using a manual parameter adjustment; see Figure 7. Following parameter sets have been determined for the low (Figure 7 left) impedances:

$$
k_{0}=1000 \frac{\mathrm{N}}{\mathrm{m}}, d_{0}=0.11 \frac{\mathrm{Ns}}{\mathrm{m}}, k_{1}=1300 \frac{\mathrm{N}}{\mathrm{m}}, d_{1}=0.33 \frac{\mathrm{Ns}}{\mathrm{m}}
$$

and for the high (Figure 7 right) impedances:

$$
k_{0}=4300 \frac{N}{m}, d_{0}=0.55 \frac{N s}{m}, k_{1}=5000 \frac{N}{m}, d_{1}=2 \frac{N s}{m}
$$



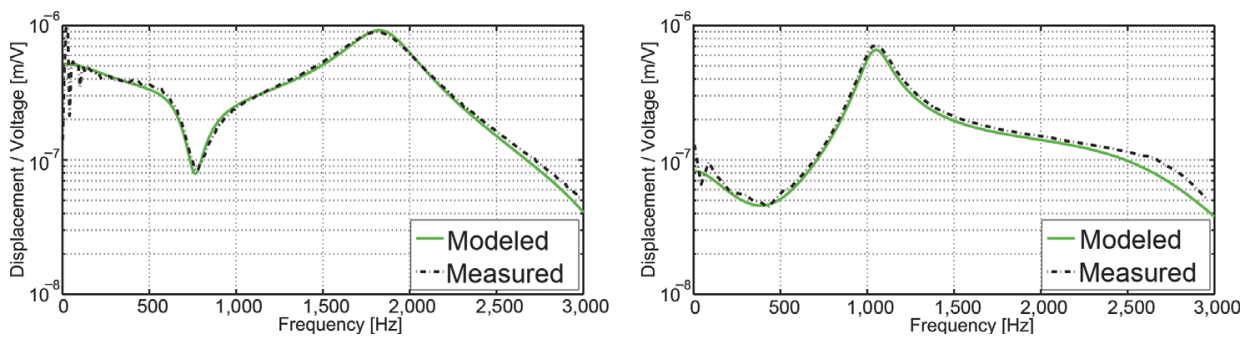

Figure 7: Real and modeled system behavior under loading conditions for a low (left) and a high (right) mechanical impedance.

\section{Actuator Optimization}

Using the analytical approach, a parametric study can be carried out to find an optimized actuator design for the display. Here the optimization is based only on the parameters variation of the middle shim layer and the actuator geometry, because the properties of the piezoelectric material usually are little influenceable by the designer.

According to the model the density has barely impact on the behavior of the loaded system. Whereas by increasing the width of the bimorph the force, which can be transferred into the skin, increases. Thus, the width should be fully exploited with respect to the available space. The Young's modulus of the middle shim layer, the middle shim layer thickness and especially the length of the bending actuator must be adapted to the load.

At this point the optimal length is to be determined for the actuator exemplarily. The procedure for the optimization of other parameters in other load conditions is similar.
The behavior for the described bimorph under load is already observed in Figure 3 and the parameters of the load model are identified. A variation of the length in the resulting actuator-load model shows that for each loading case there is an optimum in which the amplitude is greatest in the required frequency range of $0-1,000 \mathrm{~Hz}$. For the two load cases in Figure 7 the variation of the length leads to the simulation results shown in Figure 8. For the low impedance the optimal range with the largest displacement amplitudes is between 18 and $27 \mathrm{~mm}$, while for the high impedance the optimum range is located at lengths between 10 and $20 \mathrm{~mm}$. All other load cases have an optimum in between. Thus, the optimal length for our application should be in the area of overlap and this means a free bimorph length of about $20 \mathrm{~mm}$.

\section{Conclusion}

In this paper, an analytical bimorph model in the form of a compact transfer matrix is derived. The model is based
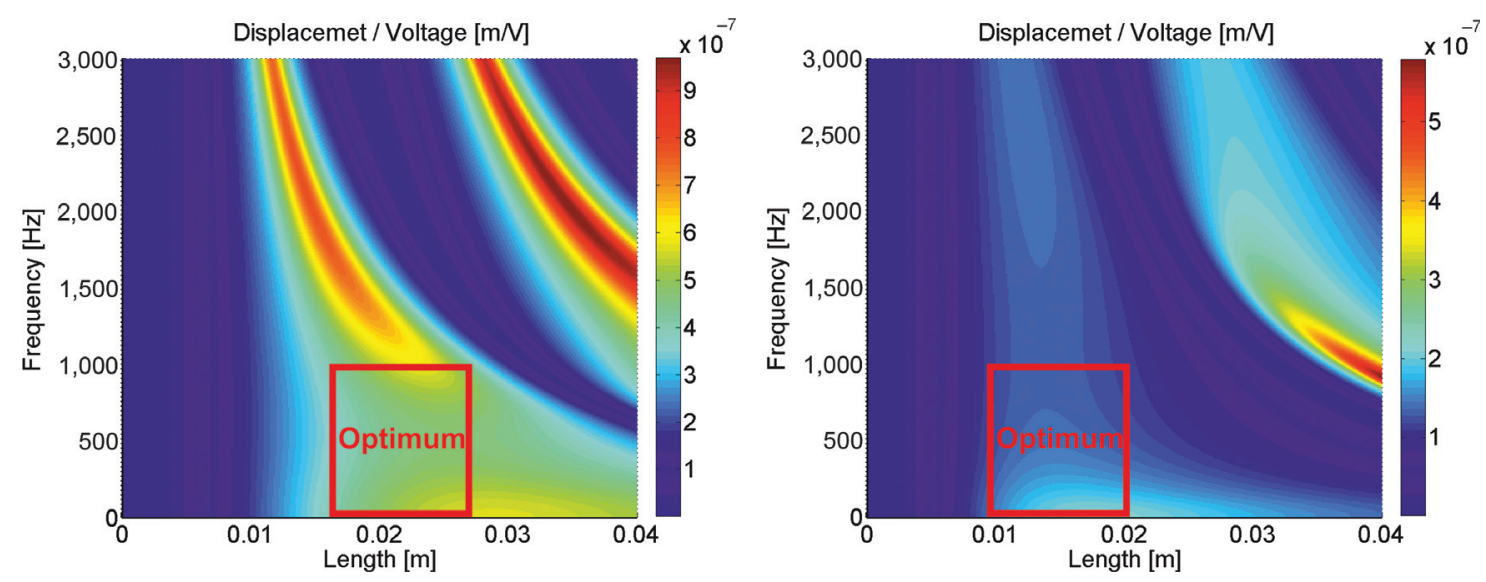

Figure 8: Modeled system behavior under loading conditions for different actuator length for a low (left) and a high (right) mechanical impedance. 
on the Timoshenko beam theory and considers the piezoelectric coupling as well as the multilayer aspects. An experimental validation of the bimorph model with the consideration of the measuring point position and the clamped actuator length was done.

In addition, a load model is derived. Therefore, an experimental investigation of the finger influence on the bimorph was carried out. So a combined actuator-load model for an analytical optimization of the bimorph could be established.

Funding: The work was supported by the German Research Foundation (DFG).

\section{Nomenclature}

$p$

$s$

l

w

$P$

$U$

$\psi$

$Q$

$(\rho l)_{\text {eff }}$ $(A G)_{\mathrm{eff}}$
Index for the piezoelectric layer Index for the middle shim layer Height Length Width Polarization direction Voltage Current Velocity Rotation angle Tilting

Moment

Force

Displacement

Cartesian coordinates

Mechanical strain

Shear

Mechanical stress

Shear stress

Dielectric charge displacement

Electrical field strength

Elastic compliance

Shear compliance

Piezoelectric constant

Dielectric constant

Superscript for constant electric field conditions

Superscript for constant stress conditions

Superscript for constant strain conditions

Rotary frequency

Density

Cross section

Electrode surface

Quality factor

Effective moment of inertia

Effective shear stiffness

$\begin{array}{ll}(E I)_{\text {eff }} & \text { Effective bending stiffness } \\ \hat{m}_{\text {eff }} & \text { Effective mass distribution } \\ C & \text { Capacity } \\ K & \text { Coupling factor } \\ \kappa & \text { Correction factor } \\ T_{1, \mathrm{~m}} & \text { Mean stress } \\ \lambda & \text { Eigenvalue } \\ n_{1}, n_{2}, a_{1}, a_{2}, a_{3}, a_{4} & \text { Abbreviations } \\ c_{0}, c_{1}, c_{2}, c_{3}, c_{4}, c_{5}, \tau, \tau^{*}, a, \beta & \text { Abbreviations } \\ \tan \delta & \text { Dielectric dissipation factor } \\ \tan \theta & \text { Piezoelectric dissipation factor } \\ j & \text { Imaginary unit } \\ Y & \text { Admittance } \\ N & \text { Normal force } \\ Z_{L} & \text { Translational impedance } \\ Z_{L, r} & \text { Rotational impedance } \\ k & \text { Stiffness parameter } \\ d & \text { Damping parameter } \\ k_{r} & \text { Torsional rigidity } \\ d_{r} & \text { Torsional damping } \\ i & \text { Running index }\end{array}$

\section{References}

Cho, Y. S., Pak, Y. E., Han, C. S., Ha, S. K. 2000. “Five-Port Equivalent Electric Circuit of Piezoelectric Bimorph Beam.” In: Sensors and Actuators, 140-8. Elsevier.

Dietl, J.M., Wickenheiser, A. M., Garcia, E. 2010. “A Timoshenko Beam Model for Cantilevered Piezoelectric Energy Harvesters. In: Smart Materials and Structures. IOP Publishing.

Gasch, R., Knothe, K., Liebich, R. 2012. Strukturdynamik, Diskrete Systeme und Kontinua, 2. Auflage. Berlin: Springer.

Hatzfeld, C. 2013. Experimental Analysis of Human Force Perception as Design Basis for Haptic Systems. Doctoral Thesis, Technische Univerität Darmstadt.

Johnson Matthey Piezo Products GmbH. 2014. "Datasheet Piezoceramic Masses." http://www.piezoproducts.com/ fileadmin/user_upload/pdf/jm_piezoproducts_data_sheet_ piezoceramic_masses_en_15_01_2015.pdf.

Kern, T. A., Matysek, M., Meckel, O., Rausch, J., Rettig, A., Röse, A., Sindlinger, S. 2009. Entwicklung Haptischer Geräte, Ein Einstieg für Ingenieure. Berlin: Springer.

Pestel, E.C., Leckie, F.A. 1963. Matrix Methods in Elasto Mechanics. New York: McGraw-Hill.

Sollmann, H. 1981. „Berechnung von erzwungenen gedämpften Biegeschwingungen mit Übertragungsmatrizen.“ In: Technische Mechanik 2, Heft 2, 69-81. Magdeburger Verein für Technische Mechanik e.V. Magdeburg.

Standards Committee of the IEEE Ultrasonics, Ferroelectrics, and Frequency Control Society. IEEE Standard on Piezoelectricity. 1987.

Uchino, K., Hirose, S. 2001. "Loss Mechanisms in Piezoelectrics: How to Measure Different Losses Separately." In: Transactions on Ultrasonics, Ferroelectrics, and Frequency Control, 48 (1): 307-21. IEEE. 